A Note on Detecting Simple Redundancies in Linear Systems

by

R.E. Bixby

Technical Report 86-14, June 1986 



\title{
A NOTE ON DETECTING SIMPLE REDUNDANCIES IN LINEAR SYSTEMS
}

\author{
Robert E. Bixby \\ Department of Mathematical Sciences \\ Rice University \\ Houston, Texas 77251 \\ Donald K. Wagner \\ School of Industrial Engineering \\ Purdue University \\ West Lafayette, Indiana $\mathbf{4 7 9 0 7}$
}

\begin{abstract}
Two efficient algorithms are presented that, for a given linear system $A x=b$, eliminate equations that are nonzero multiples of other equations. The second algorithm runs in linear time when the entries of $A$ are $+1,-1$ or 0 .
\end{abstract}

\section{INTRODUCTION}

Let $a^{1}, a^{2} \in R^{n}$, for some $n$. If $a^{1}=\gamma a^{2}$ for $\gamma \neq 0$, then $a^{1}$ is parallel to $a^{2}$. Evidently this notion of parallel induces an equivalence relation on $R^{n}$. The equivalence classes are called parallel classes. To apply this definition to a linear system $A x=b$, denote by $a^{i}$ and $b_{i}$ the $i^{\text {th }}$ row of $A$ and $i^{t h}$ entry of $b$, respectively. Equalities $a^{i} x=b_{i}$ and $a^{k} x=b_{k}$ are then said to be parallel if the vectors $\left(a^{i}, b_{i}\right)$ and $\left(a^{k}, b_{k}\right)$ are parallel. Obviously, a subsystem of equalities obtained by deleting all but one equality from each parallel class of the given system has the same solution set. Thus, from a computational point of view, preprocessing the system to remove parallel equalities may be advantageous [TW86].

Let $l_{j}$ denote the number of nonzeros in column $j$ of a given matrix $A$. We present an $O\left(\sum l_{j} \log l_{j}\right)$ algorithm (the sum here and elsewhere being over the column indices $j$ of $\left.A\right)$ to compute the parallel classes of (the set of rows of) $A$. A closely related algorithm is given in [TW86], but with a bound of $O\left(\sum l_{j}^{2}\right)$. We also show how, when $O\left(\sum l_{j}\right)$ extra storage is available and all entries are $+1,-1$, or 0 , a standard algorithm can be used to obain a linear bound: $O\left(\sum l_{j}\right)$ 


\section{AN $O\left(\sum l_{j} \log l_{j}\right)$ ALGORITHM}

In the remainder of the paper $A$ denotes an $m \times n$ matrix. To coincide with typical linear programming practice, we assume $A$ is "stored by column." Thus, for a column $j$ of $A$, we assume that the set $L=\left\{i: a_{i j} \neq 0\right\}$ can be found in time $O(|L|)$.

The idea of the algorithm is as follows. First multiply each row of $A$ by the unique scalar so that the first nonzero entry is +1 . Then two rows are parallel if and only if they are equal. Now let $A_{j}$ denote the submatrix of $A$ obtained by deleting the columns with indices $j+1$ and higher, and suppose that the parallel classes of $A_{j}$ have been determined. That is, assume that we have computed an array PCLASS such that two rows $a^{i_{1}}$ and $a^{i_{2}}$ of $A_{j}$ are parallel if and only if PCLASS $\left[i_{1}\right]=\operatorname{PCLASS}\left[i_{2}\right]$. Observe that then $a^{i_{1}}$ and $a^{i_{2}}$ are parallel in $A_{j+1}$ if and only if (PCLASS $\left.\left[i_{1}\right], a_{i_{1}, j+1}\right)=\left(\operatorname{PCLASS}\left[i_{2}\right], a_{i_{2}, j+1}\right)$. Thus, the construction of PCLASS for $A_{j+1}$ can be carried out by comparing these pairs. To facilitate this comparison, we sort first on the PCLASS values, and then among pairs that have equal PCLASS values, on the $A$ values. After this sorting, the test can be carried out by comparing adjacent pairs.

Definition. If $S$ is a set, and $f$ is a real-valued function defined on $S$, we define the $f$-sets of $S$ to be the equivalence classes of the relation $i R j \Leftrightarrow f(i)=f(j)$.

Lemma. The $f$-sets of a set $S$ can be computed in time $O(|S| \log |S|)$.

Proof. Sort $S$ as $\left[i_{1}, \ldots, i_{k}\right]$ so that $f\left(i_{1}\right) \leq \ldots \leq f\left(i_{k}\right)$, where $k=|S|$. This takes time $O(|S| \log |S|)$. The $f$-sets can then be constructed in time $O(|S|)$.

The above lemma embodies the essential difference between the algorithm below and that in [TW86]. 


\section{Algorithm}

Input: An $m \times n$ matrix $A$ stored in column-list form. We assume that the first non-zero element in each row is +1 (see Remark (1)).

Output: An array PCLASS such that two rows $a^{i_{1}}, a^{i_{2}}$ of $A$ are parallel if and only if $\operatorname{PCLASS}\left[i_{1}\right]=\operatorname{PCLASS}\left[i_{2}\right]$.

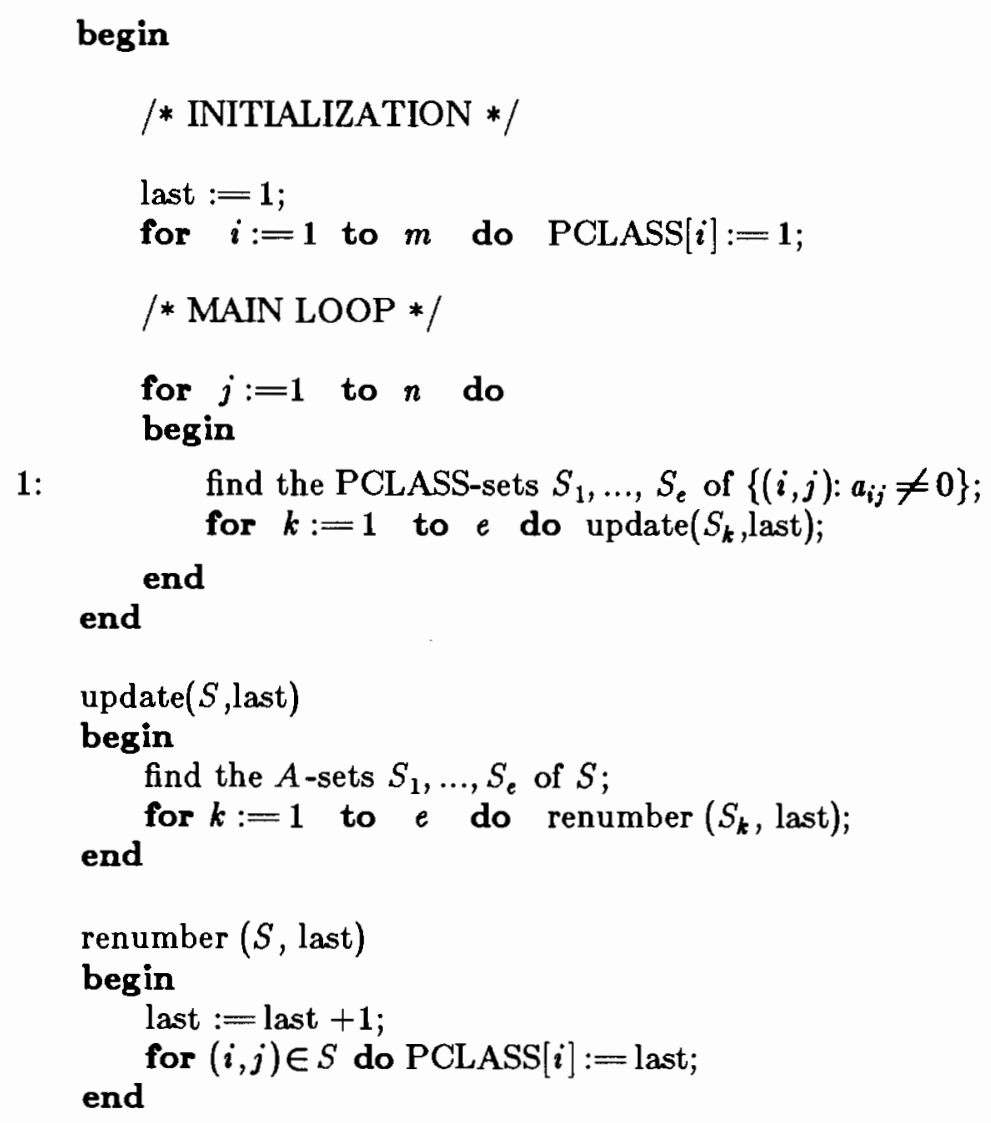

Remarks: (1) "Scaling" $A$ so that the first element of each row is +1 is easily carried out as part of 1: by keeping a "marker array" to determine the first time a row is encountered. When that happens, when a row is first encountered, we simply store the reciprocal of the corresponding entry in a "scaling array," and use this scale for further comparisons involving the row.

(2) The scaling described in Remark (1), and hence the above algorithm, is for equality systems $A x=b$. It is an easy matter to similarly deal with inequality systems $A x \leq b$. In this case we scale the first entry in each row to +1 or -1 , and insist that the scaling be by a positive scalar. Otherwise, the procedure is the same. 
(3) Note that the above algorithm may result in class numbers as large as $\sum l_{j}$. We may avoid this by keeping the class sizes in an auxiliary array and using this "size array" to determine when the old class name can be reused. In this way the class numbers are easily made to take on consecutive values between 1 and some integer not greater than $m$. It is then clear, for example, that at termination the array PCLASS can be used to "eliminate" parallel rows in time $O(m)$.

(4) In 1: we take PCLASS $[(i, j)]$ to mean PCLASS $[i]$.

Theorem. The above algorithm runs in time $O\left(\sum l_{j} \log l_{j}\right)$ and requires $O(m)$ working storage.

Proof. By virtue of the lemma, the $j t h$ iteration of the main loop takes time $O\left(l_{j} \log l_{j}\right)$. This proves the time bound. For the storage bound note that the only additional array introduced, apart from those needed to implement the sorts, is PCLASS. Since the sorts never involve any more than $m$ elements, this completes the proof.

We conjecture that the above computational bound is best possible. As justification we offer the following reasoning. Suppose we consider a dense matrix and ask for an algorithm that compares rows pairwise. Comparing two rows takes time $O(n)$, and by a standard argument we conclude that the total number of row comparisons required is $O(m \log m)$. Thus the total effort required is $O(n m \log m)$, the same as for our algorithm.

Obviously the implementation of Remark (3) does not change the contents of the above theorem. It is thus tempting to conclude that the sorts on class number can then be made into bucket sorts (since the class numbers never exceed $m$ ), and consequently carried out in time $O\left(l_{j}\right)$. Unfortunately, the actual bound may be as high as $O(m)$, because there may be as many as $m$ buckets at most stages. Note that if an acceleration to $O\left(l_{j}\right)$ could be achieved, then for special cases when the scaled $A$ matrix has only a small number of distinct values, say when $A$ originally only has the values $+1,-1$ and 0 , we could improve the overall bound for the algorithm to $O\left(\sum l_{j}\right)$, by then using bucket sort on the $A$ values. In the following section we show that, indeed, such a bound can be achieved if significant extra storage is available. 


\section{AN $O\left(\sum l_{j}\right)$ ALGORITHM FOR $0,+1,-1$ MATRICES}

Let $s=\left(s_{1}, \ldots, s_{p}\right)$ and $t=\left(t_{1}, \ldots, t_{q}\right)$ be vectors. Then $s \lesssim t$, read $s$ lexicographically less than $t$, denotes that either

1) there exists an integer $j$ such that $s_{j}<t_{j}$ and $s_{i}=t_{i}$ for all $i<j$, or

2) $\quad p \leq q$ and $s_{i}=t_{i}$ for $1 \leq i \leq p$

Lexicographically sorting a sequence of vectors $s^{1}, \ldots, s^{p}$ means to find a permutation $\pi$ such that $s^{\pi(1)} \lesssim \cdots \lesssim s^{\pi(p)}$. Given a collection of integer vectors (not necessarily of the same size), Algorithm (3.1) of [AHU74] lexicographically sorts the vectors in time $O\left(M+l_{\text {total }}\right)$, where $M$ is an upper bound on the magnitude of the entries and $l_{\text {total }}$ is sum of the lengths of the vectors.

Computing the parallel classes of $A$, where the only nonzero values in $A$ are +1 and -1 , can be reduced to this problem as follows. First, by scanning each column list once, construct a list for each row that contains all the nonzero entries in that row. Clearly, if the column lists are scanned in order, then in the list for row $i$ (say), $a_{i j}$ precedes $a_{k j}$ if $i<k$. Second, scale each such list so that the first entry is +1 . Next, append to the end of the list for row $i$ another list that contains the column indices, arranged in increasing order, of the columns that have a nonzero in row $i .^{*}$ Then two of the resulting lists, considered as vectors, are equal if and only if the corresponding rows are parallel. Thus, by lexicographically sorting the collection of vectors, the parallel classes can be found by comparing only consecutive pairs. The sorting, and therefore the computation of the parallel classes, can be done in time $O\left(\sum l_{j}\right)$ using Algorithm (3.1).

An interesting question is does there exist $O\left(\sum l_{j}\right)$ algorithm to find parallel classes when the entries of the matrix $\mathbf{A}$ are small integers? A solution to this problem could also be used on non-integer problems that have a small number distinct nonzero entries, by defining a bijection between the set of nonzero entries and a set of small integers. Such an bijection is, for example, given by any standard value-pool data structure. Note that the algorithm we have given above does not solve this problem, since the possible scaling of each row so that the first entry is a +1

*This list is easily constructed while constructing the list of nonzero entries in each row. 
may destroy the property that there are only a small number of distinct values.

We remark that it does not seem appropriate to use the above method without some restriction on the values in the matrix A. This would seem to require the use of a "stable" comparison sort algorithm at a crucial stage. Such algorithms are available with the desired [H78] bound, but are very complicated and, in practice, would not be competitive with the algorithm of the previous section. 


\section{REFERENCES}

Aho, A.V., J.E. Hopcroft and J.D. Ullman (1974). The Design and Analysis of Computer Algorithms, Addison-Wesley, Reading, Massachusetts.

Horvath, E.C. (1978). Stable Sorting in Asymptotically Optimal Time and Extra Space, JACM 25 177-199.

Knuth, D.E. (1973). Sorting and Searching, Addison-Wesley, Reading, Massachusetts.

Tomlin, J.J. and J.S. Welch (1986). Finding duplicate rows in a linear programming model. To appear in Operations Research Letters. 